\title{
CREATION OF AN OPTIMAL SHAPE OF DOMAIN USING THE TOPOLOGICAL DERIVATIVE AND BOUNDARY ELEMENT METHOD
}

\author{
Katarzyna Freus ${ }^{1}$, Sebastian Freus ${ }^{2}$ \\ ${ }^{1}$ Institute of Mathematics, Czestochowa University of Technology \\ Czestochowa, Poland \\ ${ }^{2}$ Institute of Computer and Information Science, Czestochowa University of Technology \\ Czestochowa, Poland \\ katarzyna.freus@im.pcz.pl,sebastian.freus@icis.pcz.pl
}

\begin{abstract}
The objective of this chapter is to form an optimal shape of the two-dimensional domain. This work uses an alternative manner to calculate the topological derivative based on the topological shape sensitivity method. The boundary element method is taken into account to solve the Laplace equation. The boundary of the domain is described by the Non-Uniform Rational B-Splines (NURBS) curves. In the final part of the chapter the example of an optimal shape of domain is shown.
\end{abstract}

Keywords: Laplace equation, boundary element method, topological derivative, NURBS curves

\section{Introduction}

The topological optimization process consist in removing material by creating small holes that appear within the iteration procedure. This approach allows one to find an optimal material layout of a domain, such that a cost function gives its optimum value after optimization under given constraints. The topological derivative $\left(D_{T}\right)$ shows where in the domain, the holes should be created. Wherever $D_{T}$ is low enough, a hole is formed. In the opening, the Neumann condition is imposed. The topological shape sensitivity approach is a method that allows one to calculate the topological derivative taking the total potential energy as a cost function [1-4]. After elimination of a certain amount of material, the boundary of domain must be rebuilt. This process is based on the author's algorithm that introduces some details concerning how to reconstruction the boundary and achieve the expected shape of a domain. The boundary of the domain is described by the NURBS curves which are the most popular parametric forms of curves, and they are widely used for the representation, design and data exchange of geometric information processed by computers. In order to solve the governing equation, the boundary element method (BEM) is used in its direct version. In this chapter, firstly a review of the BEM and the NURBS curves are presented. After that, the topological derivative for the Laplace equation is introduced. Next, the algorithm of rebuilding the boundary of domain is discussed. Finally, the numerical results are shown. 


\section{Boundary element method}

In this part of work a brief description of the BEM is presented. The Laplace equation supplemented by the boundary condition is taken into account $[5,6]$

$$
\begin{cases}x \in \Omega: & \lambda \nabla^{2} T(x)=0 \\ x \in \Gamma_{D}: & T(x)=T_{b} \\ x \in \Gamma_{N}: & -\lambda \frac{\partial T(x)}{\partial n}=q_{b} \\ x \in \Gamma_{R}: & -\lambda \frac{\partial T(x)}{\partial n}=\alpha\left(T(x)-T_{\infty}\right)\end{cases}
$$

where $x=\left(x_{1}, x_{2}\right)$ are the spatial coordinates, $\lambda[\mathrm{W} / \mathrm{mK}]$ is the thermal conductivity, $T(x)$ is the temperature, $\partial T / \partial n$ denotes the normal derivative and $n=\left[\cos \alpha_{1}, \cos \alpha_{2}\right]$ is the normal outward vector. $T_{b}$ and $q_{b}$ are known as the boundary temperature and heat flux, respectively. $T_{\infty}$ is the ambient temperature and $\alpha\left[\mathrm{W} / \mathrm{m}^{2} \mathrm{~K}\right]$ is the heat transfer coefficient. Equation (2) presents the boundary integral equation for problem (1)

$$
\xi \in \Gamma: \quad B(\xi) T(\xi)+\int_{\Gamma} q(x) T^{*}(\xi, x) \mathrm{d} \Gamma=\int_{\Gamma} T(x) q^{*}(\xi, x) \mathrm{d} \Gamma
$$

where $B(\xi) \in(0,1)$ is the coefficient connected with the local shape of a boundary, $\xi$ is the observation point and $q(x)=-\lambda \mathbf{n} \cdot \nabla T(x)$ is the heat flux. $T^{*}$ and $q^{*}$ are the following

$$
T^{*}(\xi, x)=\frac{1}{2 \pi \lambda} \ln \frac{1}{r}, q^{*}(\xi, x)=\frac{d}{2 \pi r^{2}}
$$

where $r$ indicates the distance between $\xi=\left(\xi_{1}, \xi_{2}\right)$ and $x=\left(x_{1}, x_{2}\right)$ while

$$
d=\left(x_{1}-\xi_{1}\right) n_{x}+\left(x_{2}-\xi_{2}\right) n_{y}
$$

$n_{x}, n_{y}$ are the directional cosines of the normal outward vector $\mathbf{n}$.

Using the linear boundary elements, Eq. (2) can be written

$$
\mathbf{G q}=\mathbf{H T}
$$

After the imposition of the known boundary condition, Eq. (5) is the following

$$
\mathbf{A X}=\mathbf{B}
$$

where $\mathbf{A}$ is the main matrix, $\mathbf{X}$ is the unknown vector and $\mathbf{B}$ is the free terms vector. Using Equation (6) allows one to find the missing boundary conditions. 


\section{Non-Uniform Rational B-Splines curves}

A $n$-th degree NURBS curve is as follows

$$
C(t)=\frac{\sum_{j=0}^{r} N_{j, n}(t) w_{j} \mathrm{P}_{j}}{\sum_{k=0}^{r} N_{k, n}(t) w_{k}}, a \leq t \leq b
$$

where $w_{j}$ are the weights, $\mathbf{P}_{j}$ are the control points forming a control polygon and $N_{j, n}(t)$ are the B-spline basis functions

$$
\begin{gathered}
N_{j, 0}(t)= \begin{cases}1, & t_{j} \leq t \leq t_{j+1} \\
0, & \text { otherwise }\end{cases} \\
N_{j, n}(t)=\frac{t-t_{j}}{t_{j+n}-t_{j}} N_{j, n-1}(t)+\frac{t_{j+n+1}-t}{t_{j+n+1}-t_{j+1}} N_{j+1, n-1}(t)
\end{gathered}
$$

prescribed for the set of nodes

$$
T=\left\{a, \ldots, a, t_{n+1}, t_{n+2}, \ldots, t_{m-(n+1)}, b, . ., b\right\}
$$

at the same time the values $a$ and $b$ appear $n+1$ times. It should be mentioned that the number of control points equals $r+1$ and corresponds to the number of nonzero basis functions. Further details can be found in [7].

\section{Topological derivative}

The topological derivative for the Laplace equation is taken into account. A small hole of radius $\varepsilon$ is created in the inside of the domain $\Omega$. The concept of the topological derivative consists in determining the sensitivity of a given cost function (total potential energy) when the size of this hole is changed. Equation (10) presents the local value of the $D_{T}[1-4]$

$$
D_{T}(x)=\lim _{\varepsilon \rightarrow 0} \frac{\psi\left(\Omega_{\varepsilon}\right)-\psi(\Omega)}{f(\varepsilon)}
$$

where $\psi(\Omega)$ and $\psi\left(\Omega_{\varepsilon}\right)$ are the cost functions calculated for the original $\Omega$ and the new domain $\Omega_{\varepsilon}$, respectively, and $f$ is a regularizing function. In order to obtain the $D_{T}$, the topological - shape sensitivity method, proposed in [1], is used 


$$
D_{T}(x)=\lim _{\substack{\varepsilon \rightarrow 0 \\ \delta \varepsilon \rightarrow 0}} \frac{\psi\left(\Omega_{\varepsilon+\delta \varepsilon}\right)-\psi\left(\Omega_{\varepsilon}\right)}{f(\varepsilon+\delta \varepsilon)-f(\varepsilon)}
$$

where $\delta \varepsilon$ is a small perturbation on the radius of the hole.

It is worth mentioning that Equations (10) and (11) are equivalent [1].

In this part of work, the topological derivative for the steady state heat transfer will be discussed. Let $\Omega_{\varepsilon}$ be the domain with a small hole. The following problem is taken into account [1-4]

$$
\begin{cases}x \in \Omega_{\varepsilon}: & \lambda \nabla^{2} T_{\varepsilon}(x)=0 \\ x \in \Gamma_{D}: & T_{\varepsilon}(x)=T_{b} \\ x \in \Gamma_{N}: & -\lambda \frac{\partial T_{\varepsilon}(x)}{\partial n}=q_{b} \\ x \in \Gamma_{R}: & -\lambda \frac{\partial T_{\varepsilon}(x)}{\partial n}=\alpha\left(T_{\varepsilon}(x)-T_{\infty}\right) \\ x \in \mathrm{H}_{\varepsilon}: & -\lambda \frac{\partial T_{\varepsilon}(x)}{\partial n}=q_{b}^{\varepsilon}\end{cases}
$$

where on the holes $\mathrm{H}_{\varepsilon}$ created via $D_{T}$, the Neumann boundary condition is given. Problem (13) can be written in the variational form with a test function $\phi$. Find $T_{\varepsilon}$ such that

$$
\int_{\Omega_{\varepsilon}} \lambda \nabla T_{\varepsilon} \cdot \nabla \phi_{\varepsilon} d \Omega_{\varepsilon}+\int_{\Gamma_{R}} \alpha T_{\varepsilon} \phi_{\varepsilon} d \Gamma_{R}=\int_{\Gamma_{R}} \alpha T_{\infty} \phi_{\varepsilon} d \Gamma_{R}-\int_{\Gamma_{N}} q_{b} \phi_{\varepsilon} d \Gamma_{N}-\int_{\mathrm{H}_{\varepsilon}} q_{b}^{\varepsilon} \phi_{\varepsilon} d \mathrm{H}_{\varepsilon}
$$

For the perturbed configuration, expression (13) is as follows

$$
\begin{gathered}
\int_{\Omega_{\varepsilon+\delta \varepsilon}} \lambda \nabla T_{\varepsilon+\delta \varepsilon} \cdot \nabla \phi_{\varepsilon+\delta \varepsilon} d \Omega_{\varepsilon+\delta \varepsilon}+\int_{\Gamma_{R}} \alpha T_{\varepsilon+\delta \varepsilon} \phi_{\varepsilon+\delta \varepsilon} d \Gamma_{R}=\int_{\Gamma_{R}} \alpha T_{\infty} \phi_{\varepsilon+\delta \varepsilon} d \Gamma_{R}+ \\
-\int_{\Gamma_{N}} q_{b} \phi_{\varepsilon+\delta \varepsilon} d \Gamma_{N}-\int_{\mathrm{H}_{\varepsilon+\delta \varepsilon}} q_{b}^{\varepsilon} \phi_{\varepsilon+\delta \varepsilon} d \mathrm{H}_{\varepsilon+\delta \varepsilon}
\end{gathered}
$$

Taking into account the total potential energy [1], the cost function $\psi\left(\Omega_{\varepsilon}\right)$ for problem (12) can be written as

$$
\begin{gathered}
\psi\left(\Omega_{\varepsilon}\right)=\frac{1}{2}\left(\int_{\Omega_{\varepsilon}} \lambda \nabla T_{\varepsilon} \cdot \nabla T_{\varepsilon} d \Omega_{\varepsilon}+\int_{\Gamma_{R}} \alpha T_{\varepsilon}^{2} d \Gamma_{R}\right)-\int_{\Gamma_{R}} \alpha T_{\infty} T_{\varepsilon} d \Gamma_{R}+ \\
+\int_{\Gamma_{N}} q_{b} T_{\varepsilon} d \Gamma_{N}+\int_{\mathrm{H}_{\varepsilon}} q_{b}^{\varepsilon} T_{\varepsilon} d \mathrm{H}_{\varepsilon}
\end{gathered}
$$


The optimization problem can be expressed as the minimization of equation (15) with the variational formulation (Eq. (13) and (14)) as constraints. These equations allow one to compute topological derivative (11) and finally lead up to the following formula

$$
D_{T}(x)=\lambda \nabla T \cdot \nabla T
$$

where $T$ is the solution of the original problem (without a hole).

In this work, the gradient $\nabla T$ is calculated by differentiating the integral equation

$$
\xi \in \Omega: \quad T(\xi)=\int_{\Gamma} T(x) q^{*}(\xi, x) \mathrm{d} \Gamma-\int_{\Gamma} q(x) T^{*}(\xi, x) \mathrm{d} \Gamma
$$

with respect to the internal points, so

$$
\xi \in \Omega: \quad \frac{\partial T(\xi)}{\partial x_{i}}=\int_{\Gamma} T(x) \frac{\partial q^{*}(\xi, x)}{\partial x_{i}} \mathrm{~d} \Gamma-\int_{\Gamma} q(x) \frac{\partial T^{*}(\xi, x)}{\partial x_{i}} \mathrm{~d} \Gamma
$$

where $T^{*}$ and $q^{*}$ are given by formulas (3).

\section{Numerical methodology}

To obtain an optimal shape of the domain, the iterative procedure is used. First, the initial domain described by the NURBS curves is provided. Then, using the BEM, the problem is solved. Next, the points with the lowest values of the topological derivative are chosen. On the selected points hexagonal holes are created. Finally, a given stop criterion is checked and the boundary of the domain is rebuilt. The iterative process is repeated until the stop criterion is obtained. It is important to mention that the material volume is checked and removed after each iteration until an expected value is obtained. The elimination process of material is halted when

$$
\operatorname{vol}\left(\Omega^{\text {final }}\right)=\beta \cdot \operatorname{vol}\left(\Omega^{\text {initial }}\right)
$$

where $\beta$ presents a determined percentage of material to be eliminated.

It is worth commenting about some details regarding the reconstruction of the boundary of the new domain. In each iteration, on the selected points, the material is eliminated by inserting a hole with the centre point $P_{0}=\left(x_{0}, y_{0}\right)$. The area of holes should be equal to about $2 \%$ of the area of the whole domain. The boundary of the openings is divided into 6 linear boundary elements (see Fig. 1). To automate the process, it is assumed that the side length of a regular hexagon is approximately equal to the length of the boundary element $\left(d_{\mathrm{s}}\right)$. 


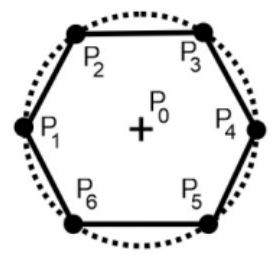

Fig. 1. Hexagonal hole

Coordinates of the hexagon vertices are the following:

$$
\begin{aligned}
& P_{1}=\left(-r+x_{0}, y_{0}\right) \\
& P_{2}=\left(-r / 2+x_{0}, h+y_{0}\right) \\
& P_{3}=\left(r / 2+x_{0}, h+y_{0}\right) \\
& P_{4}=\left(r+x_{0}, y_{0}\right) \\
& P_{5}=\left(r / 2+x_{0},-h+y_{0}\right) \\
& P_{6}=\left(-r / 2+x_{0},-h+y_{0}\right)
\end{aligned}
$$

where $r$ is a radius of the hole, while $h=r \sqrt{3} / 2$. For each node formed by the regular hexagon vertices $P_{i}=\left(x_{i}^{P}, y_{i}^{P}\right), i=1,2, \ldots 6$ the nearest boundary node is searched using the formula

$$
d_{i}=\sqrt{\left(x_{i}^{P}-x_{j}^{W}\right)^{2}+\left(y_{i}^{P}-y_{j}^{W}\right)^{2}}, \quad i=1,2, \ldots, 6, j=1,2, \ldots, n
$$

where $W_{j}=\left(x_{j}^{W}, y_{j}^{W}\right)$ and $n$ are the coordinates and the number of the boundary nodes, respectively. Next, the following criterion is considered (the so-called "contact criterion")

$$
d_{i} \leq 0.75 d_{s}
$$

If criterion (22) is satisfied, then it is assumed that the $i$-th the hexagon node keeps in contact with the $j$-th the boundary node.

Information about the number of nodes that satisfy criterion (22) or not, is stored in two separate matrices: CM (contact matrix) or NCM (not contact matrix). The matrix $\mathbf{C M}$ also includes knowledge about the distance of nodes and the type of boundary conditions in these nodes. For example, in Figure 2 nodes 2 and 34, 3 and 35 satisfy criterion (22) and 1 and 33, 4 and 36 do not satisfy. Nodes 33, 1, 6, 5,4 and 36 must be spliced to create the new outline of the domain. 


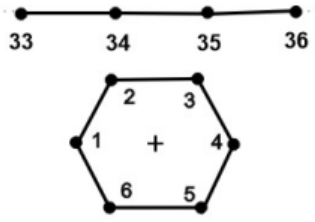

Fig. 2

If the matrix $\mathbf{C M}$ is empty (neither of the nodes is in contact) then we can create a new hole prescribing the boundary condition on its boundary (see Fig. 3). Now, we discuss some cases concerning connecting the hexagon nodes with the boundary ones. An analysis is conducted taking into account the successive nodes of the hexagon. The first case is shown in Figure 2. An increase is noticed in the numbering of boundary nodes and an increase in the numbering of hole nodes. The contact criterion is satisfied for 2 and 34, 3 and 35 nodes. In Figure 4, the numbering of hole nodes is increasing while the numbering of the boundary ones is decreasing. The nodes 5 and 3, 6 and 2 fulfill criterion (22).

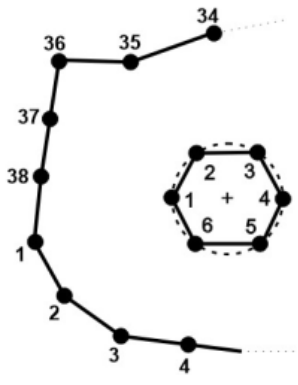

Fig. 3

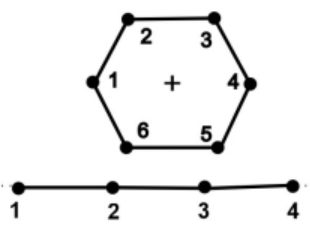

Fig. 4

Further considerations involve doing a sorting of the nodes that satisfy the contact criterion. The rows of the matrix are sorted taking into account the numbering of the boundary nodes. It is worth noting that the first column contains the hexagon's node number and the second one the node number of the boundary. For instance, two columns of the $\mathbf{C M}$ before sorting are the following (see Fig. 4).

$$
\left[\begin{array}{ll}
5 & 3 \\
6 & 2
\end{array}\right]
$$

and after sorting

$$
\left[\begin{array}{ll}
6 & 2 \\
5 & 3
\end{array}\right]
$$

Sometimes to obtain the correct sorting we need the introduce the so-called substitute numbering. It is done when both the first and the last node of the hexagon 
satisfy criterion (22). To the nodes numbered from 1, we should add 10 (this means that we replace 1 by 11,2 by 12 , etc.).

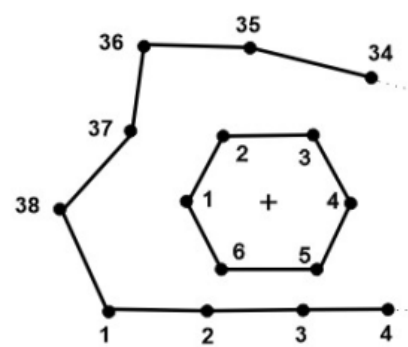

Fig. 5

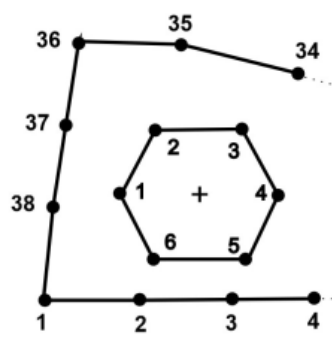

Fig. 6

For example, taking Figure 5, the contact matrix is the following

$$
\mathbf{C M}=\left[\begin{array}{cc}
5 & 3 \\
6 & 2 \\
1 & 37 \\
2 & 37
\end{array}\right]
$$

Next, in the third column of this matrix, the substitute numbering is introduced. Hence

$$
\mathbf{C M}=\left[\begin{array}{ccc}
5 & 3 & 5 \\
6 & 2 & 6 \\
1 & 37 & 11 \\
2 & 37 & 12
\end{array}\right]
$$

Since two vertices of the hexagon keep in contact with the same boundary node, the procedure of a two-level sort is used. Firstly, matrix (10) is ordered ascendingly taking into account the boundary node number and then descendingly in regard to the third substitute column. Finally, the matrix $\mathbf{C M}$ is of the form

$$
\mathbf{C M}=\left[\begin{array}{ccc}
6 & 2 & 6 \\
5 & 3 & 5 \\
2 & 37 & 12 \\
1 & 37 & 11
\end{array}\right]
$$

In the case when the boundary nodes contact, including the first and the last boundary node simultaneously, then the CM is divided into two of the following matrices 


$$
\mathbf{C M}_{1}=\left[\begin{array}{lll}
6 & 2 & 6 \\
5 & 3 & 5
\end{array}\right] \quad \mathbf{C M}_{2}=\left[\begin{array}{lll}
2 & 37 & 12 \\
1 & 37 & 11
\end{array}\right]
$$

The next step is finding a minimum and maximum number of the hexagon node and the boundary node in matrices $\mathbf{C M}_{1}$ and $\mathbf{C M}_{2}$.

$$
\begin{array}{ll}
\min _{\text {for } k=2}\left(\mathbf{C M}_{1}\right)=2 & \min _{\text {for } k=2}\left(\mathbf{C M}_{2}\right)=37 \\
\min _{\text {for } k=3}\left(\mathbf{C M}_{1}\right)=5 & \min _{\text {for } k=3}\left(\mathbf{C M}_{2}\right)=11 \\
\max _{\text {for } k=2}\left(\mathbf{C M}_{1}\right)=3 & \max _{\text {for } k=2}\left(\mathbf{C M}_{2}\right)=37 \\
\max _{\text {for } k=3}\left(\mathbf{C M}_{1}\right)=6 & \max _{\text {for } k=3}\left(\mathbf{C M}_{2}\right)=12
\end{array}
$$

where $\mathrm{k}$ is the appropriate number of a column in the matrices. The nodes that are not in contact create a new contour of domain. It is achieved by searching for a minimum and maximum of the hexagon nodes. The boundary nodes from 1 to $\min \left(\mathbf{C M}_{1}\right)$ and from 1 to $\min \left(\mathbf{C M}_{2}\right)$ are removed. The different case is when all of the hexagon nodes keep in contact (see Fig. 6). Then a closed contour is obtained using the new boundary element by connecting boundary nodes 4 and 34 .

\section{Numerical example and results}

This section presents the solution of problem (12). It is assumed that $\lambda=$ $=300 \mathrm{~W} / \mathrm{mK}$, the heat transfer coefficient is $\alpha=10 \mathrm{~W} /\left(\mathrm{m}^{2} \mathrm{~K}\right)$ and the ambient temperature equals $T_{\infty}=20^{\circ} \mathrm{C}$. The position of control points are the following: $\mathbf{P}_{0}=(0,0), \mathbf{P}_{1}=(0.02,0), \mathbf{P}_{2}=(0.02,0.015), \mathbf{P}_{3}=(0.035,0.015), \mathbf{P}_{4}=(0.05,0.015)$, $\mathbf{P}_{5}=(0.05,0), \quad \mathbf{P}_{6}=(0.104,0), \quad \mathbf{P}_{7}=(0.104,0.011), \quad \mathbf{P}_{8}=(0.115,0.011)$, $\mathbf{P}_{9}=(0.126,0.011), \quad \mathbf{P}_{10}=(0.126,0), \quad \mathbf{P}_{11}=(0.15,0), \quad \mathbf{P}_{12}=(0,0.035)$, $\mathbf{P}_{13}=(0.075,0.075), \mathbf{P}_{14}=(0.035,0.075)$. Figure 8 shows the dimensions and boundary of domain considered.

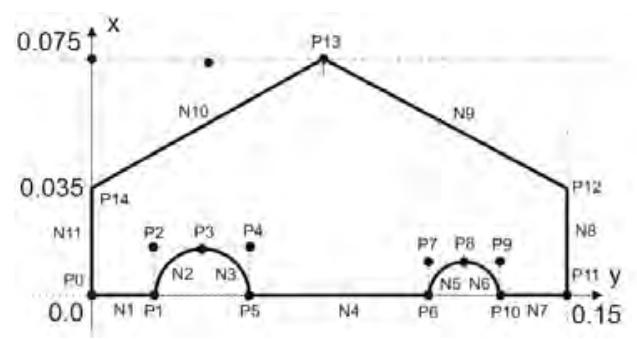

Fig. 7. Position of control points

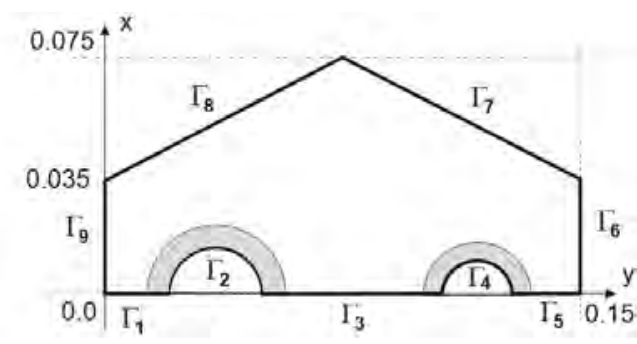

Fig. 8. Boundary of domain 
The boundary of the region is represented by the parametric curves as follows:

$\Gamma_{1}: \quad \mathrm{N} 1(t)=(1-t) \mathrm{P}_{0}+t \mathrm{P}_{1}, \quad t \in[0,1]$

$\Gamma_{2}: \begin{cases}\mathrm{N} 2(t)=\frac{(1-t)^{2} w_{0} \mathrm{P}_{3}+2 t(1-t) w_{1} \mathrm{P}_{2}+t^{2} w_{2} \mathrm{P}_{1}}{(1-t)^{2} w_{0}+2 t(1-t) w_{1}+t^{2} w_{2}}, & w_{0}=w_{1}=1, w_{2}=2 \\ \mathrm{~N} 3(t)=\frac{(1-t)^{2} w_{0} \mathrm{P}_{5}+2 t(1-t) w_{1} \mathrm{P}_{4}+t^{2} w_{2} \mathrm{P}_{3}}{(1-t)^{2} w_{0}+2 t(1-t) w_{1}+t^{2} w_{2}}, & w_{0}=w_{1}=1, w_{2}=2\end{cases}$

$\Gamma_{3}: \quad \mathrm{N} 4(t)=(1-t) \mathrm{P}_{5}+t \mathrm{P}_{6}, t \in[0,1]$

$\Gamma_{4}: \begin{cases}\mathrm{N} 5(t)=\frac{(1-t)^{2} w_{0} \mathrm{P}_{8}+2 t(1-t) w_{1} \mathrm{P}_{7}+t^{2} w_{2} \mathrm{P}_{6}}{(1-t)^{2} w_{0}+2 t(1-t) w_{1}+t^{2} w_{2}}, & w_{0}=w_{1}=1, w_{2}=2 \\ \mathrm{~N} 6(t)=\frac{(1-t)^{2} w_{0} \mathrm{P}_{10}+2 t(1-t) w_{1} \mathrm{P}_{9}+t^{2} w_{2} \mathrm{P}_{8}}{(1-t)^{2} w_{0}+2 t(1-t) w_{1}+t^{2} w_{2}}, & w_{0}=w_{1}=1, w_{2}=2\end{cases}$

$\Gamma_{5}: \quad \mathrm{N} 7(t)=(1-t) \mathrm{P}_{10}+t \mathrm{P}_{11}, \quad t \in[0,1]$

$\Gamma_{6}: \quad \mathrm{N} 8(t)=(1-t) \mathrm{P}_{11}+t \mathrm{P}_{12}, \quad t \in[0,1]$

$\Gamma_{7}: \quad \mathrm{N} 9(t)=(1-t) \mathrm{P}_{12}+t \mathrm{P}_{13}, \quad t \in[0,1]$

$\Gamma_{8}: \quad \mathrm{N} 10(t)=(1-t) \mathrm{P}_{13}+t \mathrm{P}_{14}, t \in[0,1]$

$\Gamma_{9}: \quad \mathrm{N} 8(t)=(1-t) \mathrm{P}_{14}+t \mathrm{P}_{0}, \quad t \in[0,1]$

The gray area (see Fig. 8) will not be perturbed (this is the structural part of the problem). On the boundary $\Gamma_{6} \cup \Gamma_{7} \cup \Gamma_{8} \cup \Gamma_{9}$, the Robin condition is considered, on $\Gamma_{2}$ the temperature $T_{b}=90^{\circ} \mathrm{C}$ is given while on $\Gamma_{4}, T_{b}=50^{\circ} \mathrm{C}$ is accepted. On the remaining parts of the boundary $q_{b}=0$ is prescribed. The initial boundary was divided into 80 linear boundary elements and the grid of 74 internal nodes was used.

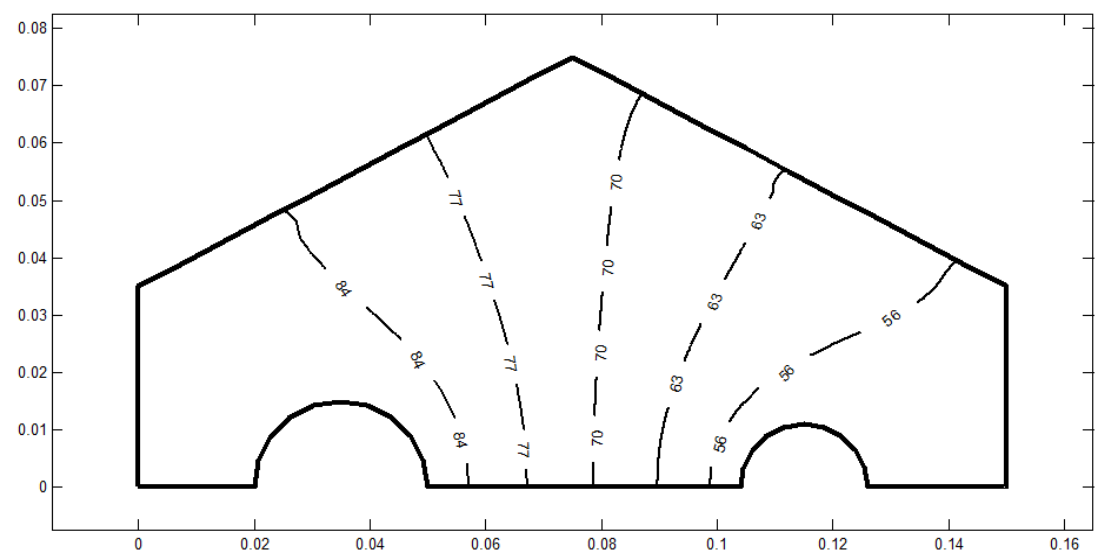

Fig. 9. Temperature distribution 
Figure 9 presents the temperature distribution while Figure 10 illustrates the topological derivative calculated in the first iteration $(k=1)$. Figure 11 presents the domain after the first iteration. In the opening, $q_{b}^{\varepsilon}=0$ was prescribed. Holes with $r=0.002$ were used, and during each iteration $2 \%$ of material was removed. The iterative procedure was stopped when $60 \%$ of material from the initial domain was eliminated. Figure 12 presents the final solution.

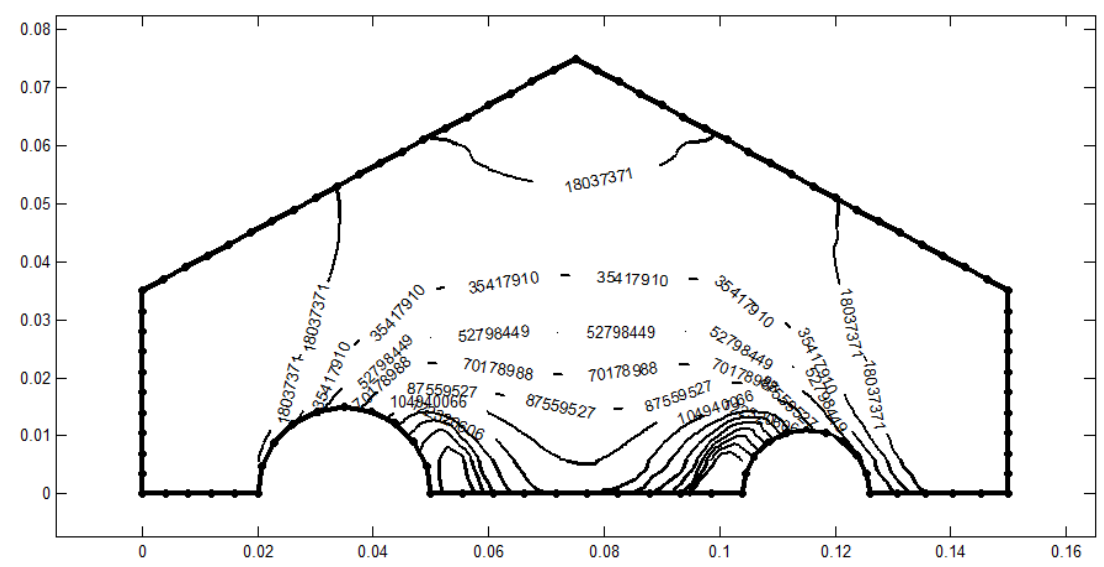

Fig. 10. Topological derivative at $k=1$

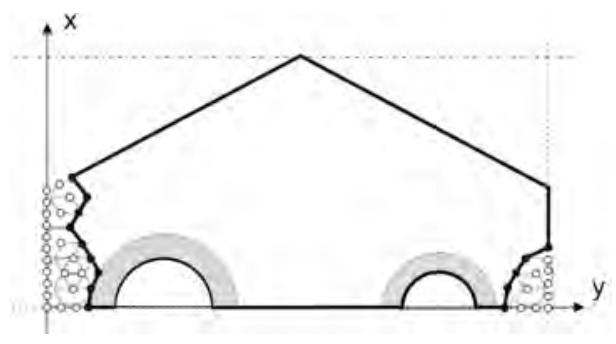

Fig. 11. Domain after the first iteration

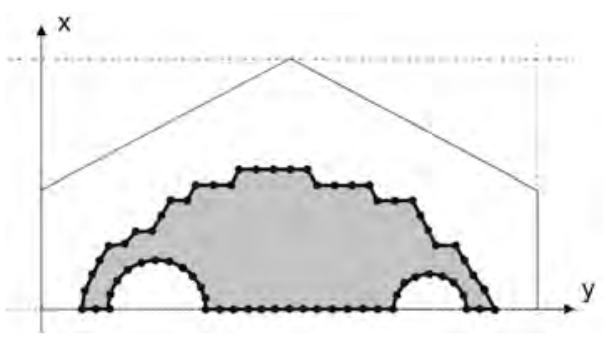

Fig. 12. Final result

\section{Conclusions}

The present work confirms the effectiveness of the proposed algorithm of the rebuilding boundary and the boundary element method coupled with the topological derivative, for creating an optimal shape of the domain. The boundary of domain was described by NURBS curves. The topological shape sensitivity method was used to obtain information about the position, where the holes should be inserted. After applying the iterative process, an optimal shape of the domain was found. In order to check the correctness of the method, the example of computation was solved. The results of the study show good agreement with the available literature. 


\section{References}

[1] Navotny A.A., Feijoo R.A., Taroco E., Padra C., Topological-shape sensitivity analysis, Comput. Methods Appl. Mech. Eng. 2003, 192, 803-829.

[2] Marczak R.J., Topology optimization and boundary elements - a preliminary implementation for linear heat transfer, Engineering Analysis with Boundary Elements 2007, 31, 793-802.

[3] Anflor C.T.M., Marczak R.J., Topological sensitivity analysis for two-dimensional heat transfer problems using the Boundary Element Method, Optimization of Structures and Components Advanced Structured Materials 2013, 43, 11-33.

[4] Anflor C., Marczak R.J., A boundary element approach for shape and topology design in orthotropic heat transfer problems, Mecanica Computacional vol. XXVII, 2473-2486, San Luis, Argentina, 10-13 Noviembre 2008.

[5] Brebbia C.A., Dominguez J., Boundary Elements, An Introductory Course, CMP, McGraw-Hill Book Company, London 1992.

[6] Majchrzak E., Boundary Element Method in Heat Transfer, Publ. of the Techn. Univ. of Czest., Czestochowa 2001 (in Polish).

[7] Piegl L., Tiller W., The NURBS Book, Springer, 1995. 